\title{
Regular and Periodic Tachyon Kinks
}

\author{
D. Bazeia, R. Menezes, and J.G. Ramos \\ Departamento de Física, Universidade Federal da Paraíba \\ Caixa Postal 5008, 58051-970 João Pessoa PB, Brazil
}

\begin{abstract}
We search for regular tachyon kinks in an extended model, which includes the tachyon action recently proposed to describe the tachyon field. The extended model that we propose adds a new contribution to the tachyon action, which allows obtaining stable tachyon kinks of regular profile, which may appropriately lead to the singular kink found by Sen sometime ago. Also, under specific conditions we may find periodic array of kink-antikink configurations.
\end{abstract}

PACS: 11.25.Uv; Keywords: tachyons, kinks 


\section{Contents}

1. Introduction

2. Generalities 2

3. Specific models 1

3.1 Type-I models 1

3.2 Type-II models

3.3 Type-III models

4. Periodic solutions 6

5. Ending comments 8

\section{Introduction}

String theory is perhaps the most plausible candidate for a relativistic theory to describe the electromagnetic, weak, strong, and gravitational interactions altogether. It engenders a very rich structure which includes stable or BPS and unstable or non-BPS branes. In the last case, instabilities of non-BPS branes are marked by the presence of tachyon fields, whose dynamics are directly related to the process in which non-BPS branes decay into BPS branes — see e.g. Refs. [1, 2, 3, 4, 5, 6, 7, 8].

These recent investigations on tachyons have suggested that the tachyon dynamics is described by the action

$$
S=-\int d^{p+1} x V(T) \sqrt{1+\eta^{\mu \nu} \partial_{\mu} T \partial_{\nu} T}
$$

where $T=T(\vec{x}, t)$ is the tachyon field, real, and $x^{\mu}=(t, \vec{x})$ is the position vector. Also, the Minkowski metric has signature $(-,+,+,+, \cdots)$, and $V(T)$ is the potential, which is non negative, obeys $V(T \rightarrow \pm \infty) \rightarrow 0$ and attains its global maximum at $T=0$. We consider $0 \leq V(T) \leq 1$.

In the present work, in the above action we modify the Lagrange density to the new form

$$
\widetilde{\mathcal{L}}=-V(T)\left(\sqrt{1+\eta^{\mu \nu} \partial_{\mu} T \partial_{\nu} T}-\frac{1}{\sqrt{1+r^{2} F^{2}}}\right)
$$

where $r$ is a parameter, real and positive, and $F=F\left(T, \eta^{\mu \nu} \partial_{\mu} T \partial_{\nu} T\right)$. The real parameter $r$ is introduced to control the way one goes beyond former investigations: for $F$ that behaves appropriately, the limit $r \rightarrow \infty$ gives the former action (1.1) and leads to the problem investigated by Sen in Ref. [9]; for $r>>1$ one may get to the recent investigation [10], in 
which one modifies the action (1.1) by adding a term which depends on the derivative of the tachyon field. In Ref. [10], the term included in the action is controlled by a very small parameter, and admits a perturbative investigation which very nicely leads to regular kink, and gives the singular kink of Ref. [9] in the appropriate limit.

In this work, in the modification we are introducing in (1.2) we consider functions that only depend on the tachyon field, that is, we consider $F=F(T)$ as a non negative function $T$ alone. Moreover, we suppose that $F(T)$ is limited to some interval, that is, we suppose that $0 \leq F(T) \leq 1$. As we shall show, the above model engenders specific features which are of direct interest to high energy physics. To do this, we organize the subject of this work in the sections that follow, where we study the presence of tachyon kinks of regular and periodic profile.

\section{Generalities}

We use the modified model to examine the energy corresponding to static configuration $T=T(\vec{x})$. We get

$$
E=\int d^{p} x V(T)\left(\sqrt{1+\partial_{i} T \partial^{i} T}-\frac{1}{\sqrt{1+r^{2} F^{2}}}\right)
$$

We investigate stability of static solutions with the Derrick-Hobart theorem [11, 12, 13, 14] — see also Ref. [10]. We change $T(\vec{x}) \rightarrow T^{\lambda}(\vec{x})=T(\lambda \vec{x})$ to get to the condition for stability of the solution $T(\vec{x})$

$$
\frac{p+(p-1) \partial_{i} T \partial^{i} T}{\sqrt{1+\partial_{i} T \partial^{i} T}}=\frac{p}{\sqrt{1+r^{2} F^{2}}}
$$

The case of a single spatial dimension is special. Here the above condition reduces to the simpler form $T^{2}(x)=r^{2} F^{2}(T)$, or better

$$
T^{\prime}(x)= \pm r F(T)
$$

where the prime stands for derivative with respect to $x$. We note that the above Eq. (2.3) reproduces first-order differential equations that appear in the bosonic sector of supersymmetric field theory described a single chiral superfield; see, e.g., Ref. 115].

Let us now investigate the equation of motion that follows from the modified action. It can be written in the form

$$
\frac{1}{\sqrt{1+\partial_{\mu} T \partial^{\mu} T}}\left(\frac{d V}{d T}-V \partial_{\mu} \partial^{\mu} T+V \frac{\partial^{\mu} T \partial^{\nu} T \partial_{\mu} \partial_{\nu} T}{1+\partial_{\mu} T \partial^{\mu} T}\right)=\frac{1}{\sqrt{1+r^{2} F^{2}}}\left(\frac{d V}{d T}-\frac{r^{2} F V}{1+r^{2} F^{2}} \frac{d F}{d T}\right)
$$

In the case $p=1$, the static field obeys

$$
\frac{1}{T^{\prime}} \frac{d}{d x}\left(\frac{V}{\sqrt{1+T^{\prime 2}}}-\frac{V}{\sqrt{1+r^{2} F^{2}}}\right)=0
$$

This equation is solved by $T^{\prime} \rightarrow \infty$, which are stable solutions of the equation (2.3) in the limit $r \rightarrow \infty$, which leads to the case first investigated by Sen in [9], giving rise to the 
stable but singular tachyon kinks

$$
T_{S}^{ \pm}(x)= \begin{cases} \pm \infty & \text { for } x>0 \\ 0 & \text { for } x=0 \\ \mp \infty & \text { for } x<0\end{cases}
$$

There are other solutions, which obey

$$
T^{\prime 2}=\frac{1}{\left(\frac{V_{0}}{V}+\frac{1}{\sqrt{1+r^{2} F^{2}}}\right)^{2}}-1
$$

where $V_{0}$ is a real constant, constrained to obey

$$
0 \leq \frac{1}{\sqrt{1+r^{2} F^{2}}}+\frac{V_{0}}{V} \leq 1
$$

The case $V_{0}=0$ reproduces the former Eq. (2.3), leading to the conclusion that the solutions of the equation of motion are stable for $V_{0}=0$.

In our model, in the case $p=1$ the unidimensional stable static solutions have energy in the form

$$
E=r^{2} \int d x \frac{V(T) F^{2}(T)}{\sqrt{1+r^{2} F^{2}(T)}}
$$

In the case $r \rightarrow \infty$ we get

$$
E_{S}=\int_{-\infty}^{\infty} d T V(T)
$$

which requires that the tachyonic potential be integrable. Another case is given by $r=0$. Stable solutions should satisfy $T^{\prime}=0$, which makes the tachyonic field constant. The energy associated to such constant configurations vanishes. The energy in (2.9) is nonnegative, and for stable solutions it varies in the interval $0 \leq E \leq E_{S}$, for functions $F(T)$ which behave appropriately.

We notice that in the modified model, in the case $p=1$ stable tachyon solutions obey $T^{\prime}=r F(T)$, and so they do not depend on the explicit form of the tachyon potential. However, the tachyon potential plays the important role of controlling the energy of the tachyon solution. This fact helps us to understand why the singular tachyons (2.6) are stable, finite energy solutions.

We also notice that in the modified model, the energy-momentum tensor $T_{\mu \nu}$ gives the energy density $T_{00}$

$$
T_{00} \equiv V(T)\left(\sqrt{1+T^{2}}-\frac{1}{\sqrt{1+r^{2} F^{2}}}\right)
$$

and pressure along the non trivial $x$ direction $\left(P_{1}=T_{11}\right)$

$$
T_{11} \equiv-\frac{V}{\sqrt{1+T^{\prime 2}}}+\frac{V}{\sqrt{1+r^{2} F^{2}}}
$$

The pressure is constant since $T_{11}^{\prime}(x)=0$ - see Eq. (2.5). We see that $T_{11}=-V_{0}$, where $V_{0}$ is the constant that we have introduced to write Eq. (2.7). Thus, the case 
$V_{0}=0$ corresponds to vanishing pressure, and gives rise to stable finite energy tachyon configurations which obey $T^{\prime}= \pm r F$. Furthermore, since $V_{0}$ must obey the constraint (2.8), we can also have two other distinct possibilities: one for $V_{0}$ positive, representing the case of negative pressure, and the other for $V_{0}$ negative, representing the case of positive pressure. We shall show below that in the case of non vanishing $V_{0}$, we must compactify the real line in order to have finite energy, and this will give rise to periodic tachyon kinks. Thus, in the modified model we shall find stable and regular tachyon kinks in an environment with vanishing pressure. And also, we shall find periodic kink-antikink array in another environment, with negative pressure. We notice from the above Eq. (2.12) that the limit $r \rightarrow \infty$, which leads to the standard tachyon action, gives rise to the case of negative pressure, and nothing more - see Ref. [16, 17].

\section{Specific models}

We investigate the case with $V_{0}=0$, which corresponds to vanishing pressure. This case gives rise to models that support stable tachyon kinks of regular profile. The energy depends on the tachyon potential, $V(T)$. Thus, we choose the tachyon potential such that $E_{S}=1$. In this case the energy of the tachyon configurations is restricted to be in the interval $0<E<1$. We may choose $V_{I}(T)=\exp \left(-\pi T^{2}\right), V_{I I a}(T)=1 / 2 \cosh ^{2}(T)$, $V_{I I b}(T)=1 / \pi \cosh (T)$, and $V_{I I I}(T)=1 / \pi\left(1+T^{2}\right)$ which identify type-I, type-II, and type-III models, all leading to unit energy. We will study these models to have a better understanding of the role of the potential for the modified tachyon action that we propose in (1.2). Specific models involving the choices $F(T)=1$ and $F(T)=1 / \cosh (T)$ will be investigated below.

\subsection{Type-I models}

We consider the case $F(T)=1$, which implies that $T^{\prime}= \pm r$, giving rise to the solutions $T_{ \pm}(x)= \pm r x$, which lead to the singular kinks (2.6) in the limit $r \rightarrow \infty$. This case reproduces the solutions of Ref. [10]. The energy corresponding to these solutions has the form

$$
E_{I}(r)=\frac{r^{2}}{\sqrt{1+r^{2}}} \int_{-\infty}^{\infty} d x V(T)
$$

Thus, for $V(T)=\exp \left(-\pi T^{2}\right)$ and for $T_{ \pm}= \pm r x$ we get

$$
E_{I}(r)=\frac{r}{\sqrt{1+r^{2}}}
$$

We use this result to get $E(r=0)=0$. This is interesting, since the limit $r \rightarrow 0$ leads to a constant tachyon configuration. Our model gives vanishing energy for trivial constant tachyon configurations at $r=0$, and unit energy for the singular kink (2.6) at the limit $r \rightarrow \infty$. The stable kink solutions $T^{ \pm}(x)= \pm r x$ are parametrized by $r$, and have energy as in Eq. (3.2), which is well-defined in the entire interval $0 \leq r \leq 1$. In Fig. [1] we plot $E_{I}(r)$ in the whole interval $r \in[0, \infty)$. 
We also consider the case of $F(T)=1 / \cosh (T)$ to get

$$
T^{\prime}= \pm \frac{r}{\cosh (T)}
$$

This equation was already solved in Ref. [18]. The solutions are $T(x)= \pm \operatorname{arcsinh}(r x)$, and we realize that the singular kink (2.6) is now very naturally recovered in the limit $r \rightarrow \infty$. The energy of the regular kinks can be written as

$$
\widetilde{E}_{I}(r)=r \int_{-\infty}^{\infty} d x \frac{e^{-\pi \operatorname{arcsinh}^{2}(x)}}{\left(1+x^{2}\right) \sqrt{1+\frac{r^{2}}{1+x^{2}}}}
$$

and depends on the parameter $r$. It vanishes for $r=0$, and converges to unit in the limit $r \rightarrow \infty$. In Fig. [1] we plot the energy density for $r \in[0, \infty)$. We note that $\widetilde{E}_{I}(r)$ is very close to $E_{I}(r)$, indicating that the choice of $F(T)$ determine no qualitative behavior.

\subsection{Type-II models}

We first consider type-IIa models, and use $F(T)=1$. The investigation is similar to the former case. The kink solutions and the energy give the very same results already obtained in the corresponding type-I model. Thus, we consider the next case: $F(T)=1 / \cosh (T)$. Here the energy changes to

$$
\widetilde{E}_{I I a}(r)=\frac{r}{2} \int_{-\infty}^{\infty} d x \frac{1}{\left(1+x^{2}\right)^{2}} \frac{1}{\sqrt{1+\frac{r^{2}}{1+x^{2}}}}
$$

It vanishes for $r=0$, and converges to unit in the limit $r \rightarrow \infty$. In Fig. [1] we plot the energy $\widetilde{E}_{I I a}(r)$ in the entire interval $r \in[0, \infty)$. We note that $\widetilde{E}_{I I a}(r)$ is very close to $\widetilde{E}_{I}(r)$, suggesting that the specific choice of the tachyonic potential seems to determine no qualitative behavior.

We also consider type-IIb models, with the potential $1 / \pi \cosh (T)$. For $F(T)=1$ we get the very same result already obtained in the former case. For $F(T)=1 / \cosh (T)$, the investigation is slightly modified, with the energy changing to

$$
\widetilde{E}_{I I b}(r)=\frac{r}{\pi} \int_{-\infty}^{\infty} d x \frac{1}{\left(1+x^{2}\right)^{3 / 2}} \frac{1}{\sqrt{1+\frac{r^{2}}{1+x^{2}}}}
$$

In Fig. [1] we also plot $\widetilde{E}_{I I b}$ as a function of $r$. We see that it is similar to $\widetilde{E}_{I I a}$, showing that tachyon potentials of the form $1 / \cosh (T)$ and $1 / \cosh ^{2}(T)$ give very similar results.

\subsection{Type-III models}

Again, we first consider the case $F(T)=1$. The investigation is similar to the former cases, and both the kink solutions and the energy give the very same results already obtained, Thus, we consider the next case of $F(T)=1 / \cosh (T)$. The kink solutions are the same, but the energy changes to

$$
\widetilde{E}_{I I I}(r)=\frac{r}{\pi} \int_{-\infty}^{\infty} d x \frac{\left[1+\operatorname{arcsinh}^{2}(x)\right]^{-1}}{\left(1+x^{2}\right) \sqrt{1+\frac{r^{2}}{1+x^{2}}}}
$$


It vanishes for $r=0$, and converges to unit in the limit $r \rightarrow \infty$. However, the convergence is very slow, due to the specific form of the tachyon potential in this case. In Fig. [1] we also plot $\widetilde{E}_{I I I}$ as a function of $r$. Its behavior is now distinct from the others, and this shows that the inverse power behavior of the tachyon potential leads to distinct energy behavior for tachyon kink, for $F(T) \neq 1$.

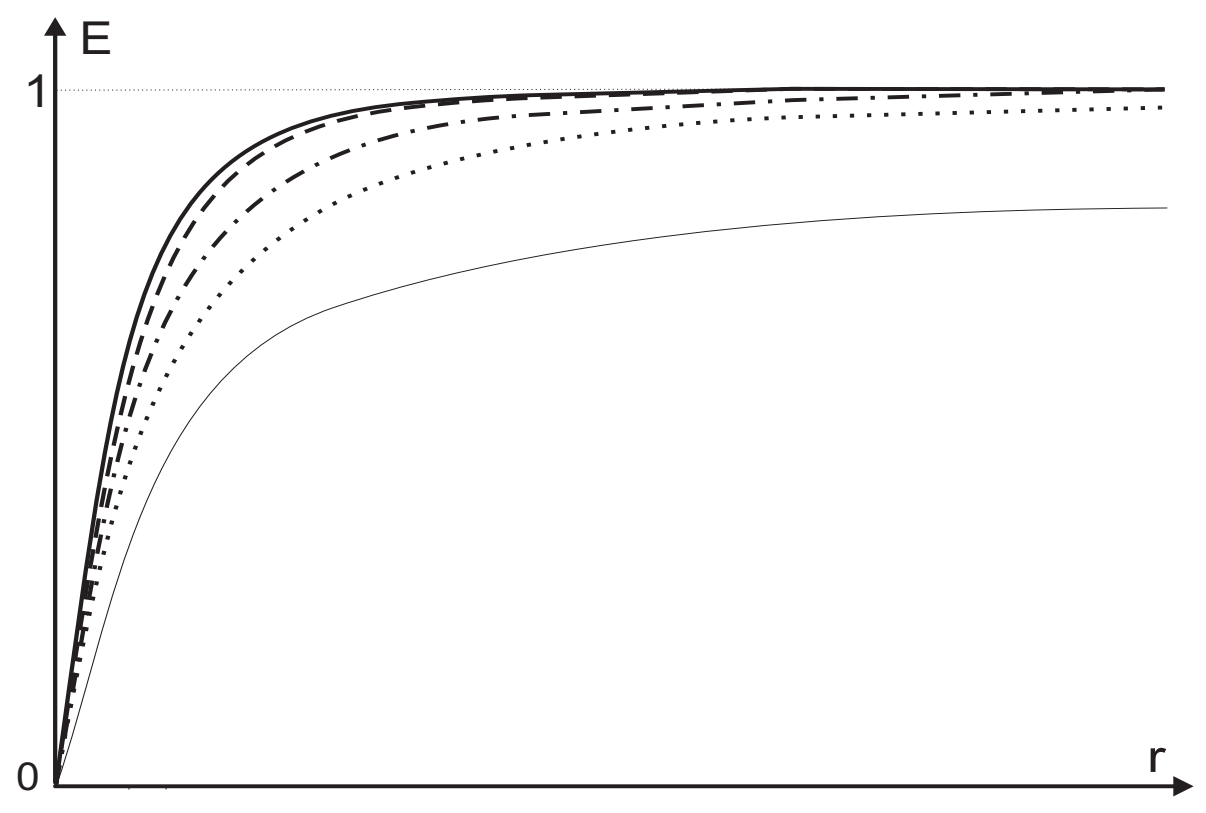

Figure 1: The energy as a function of the real parameter $r$. The thick line corresponds to $F(T)=1$, for all the models. The dashed, dash-dotted, and dotted lines correspond to $F(T)=1 / \cosh (T)$ for the type-I, type-IIa, and type-IIb models, respectively. The thin line corresponds to the type-III model, for $F(T)=1 / \cosh (T)$.

\section{Periodic solutions}

We now consider other possibilities, which appear when $V_{0} \neq 0$. As we have already seen, for $V_{0} \neq 0$ there are no stable solutions. However, we can compactify the real line to investigate static, periodic and finite energy solutions. We follow Refs. [16, 17], which has already investigated the presence of static, periodic and finite energy solutions in the model (1.1).

In order to study static, periodic and finite energy solutions in the modified model, we consider the case $V(T)=V_{I I a}(T)=1 / 2 \cosh ^{2}(T)$, and the case $F(T)=1$. We get

$$
T_{p}^{\prime 2}(x)=\frac{1}{\left[1 / \sqrt{1+r^{2}}+2 V_{0} \cosh ^{2}(T)\right]^{2}}-1
$$

The constant $V_{0}$ is now restricted to belong to an interval which depends on $r$. A specific case is $r=0$, and now we have $-1 / 2 \leq V_{0} \leq 0$, with the tachyon field constrained to live in the interval $-T_{0} \leq T \leq T_{0}$, with $T_{0}=\operatorname{arccosh} \sqrt{-1 / 2 V_{0}}$. This case corresponds to positive pressure. 
We use $r=0$ to define the function

$$
G(T)=\frac{1}{\left[1+2 V_{0} \cosh ^{2}(T)\right]^{2}}-1
$$

In this case, we notice that the points $\pm T_{0}$ are divergences for $G(T)$, and so the periodic solutions should end vertically, with divergent derivative. This behavior is not admissible. Indeed, there are no finite energy periodic solutions for $V_{0}<0$, in the case of positive pressure. We see this using the energy density to write, for $F(T)=1$, and for $r=0$ : $T_{00}(x)=-V_{0} /\left(1+V_{0} / V\right)$. We take $V=V_{I I a}$ to get

$$
T_{00}(x)=-\frac{V_{0}}{1+2 V_{0} \cosh ^{2}(T)}
$$

The constant $V_{0}$ should obey $-1 / 2 \leq V_{0} \leq 0$, and the energy density diverges at the values $\pm \operatorname{arccosh} \sqrt{-1 / 2 V_{0}}$. By the way, we notice that the energy vanishes in the limit $V_{0} \rightarrow 0$, because the choice $F(T)=1$ gives rise to tachyon kinks which obey $T^{\prime}(x)= \pm r$, and for $r=0$ we get to trivial tachyon solutions with vanishing energy.

We now consider the case $r \rightarrow \infty$, which gives $0 \leq 2 V_{0} \cosh ^{2}(T) \leq 1$. There are solutions for $0 \leq V_{0} \leq 1 / 2$, with $-\operatorname{arccosh}\left(\sqrt{1 / 2 \mathrm{~V}_{0}}\right) \leq T \leq \operatorname{arccosh}\left(\sqrt{1 / 2 \mathrm{~V}_{0}}\right)$. This case corresponds to negative pressure, and the solutions are similar to the periodic kinks found in Ref. [16, 17]. In the modified model, $V_{0}$ varies in the interval $\left[-1 / 2 \sqrt{1+r^{2}}, 1 / 2-\right.$ $\left.1 / 2 \sqrt{1+r^{2}}\right]$, which depends on $r$. For $V_{0}=0$, there are finite energy tachyon kinks which engender very nice profile, and for $V_{0}$ positive there are periodic solutions similar to the solutions found in Ref. [16, 17].

We can illustrate both cases involving positive and negative pressure with simpler models. We consider the case of a single real scalar field to write the Lagrange density

$$
\mathcal{L}=-\frac{1}{2} \partial_{\mu} \phi \partial^{\mu} \phi-\frac{1}{2}\left(\frac{d W}{d \phi}\right)^{2}
$$

where $W=W(\phi)$ is a smooth function of $\phi$. The case of periodic tachyon kinks for negative pressure is similar to the case described by

$$
W(\phi)=\frac{1}{2} \phi \sqrt{1-\phi^{2}}+\frac{1}{2} \arcsin (\phi)
$$

which requires that $\phi \in[-1,1]$. In this case the first-order equations are given by

$$
\frac{d \phi}{d x}= \pm \sqrt{1-\phi^{2}}
$$

They are solved by $\phi(x)= \pm \sin (x)$ and this requires that $x \in[-\pi / 2, \pi / 2]$.

We can make an array of kink-antikink by alternating kinks and antikinks in the real line. The energy density of each kink or antikink is $\varepsilon(x)=\cos ^{2}(x)$, which can be integrated in the interval $-\pi / 2 \leq x \leq \pi / 2$ to give $E_{k}=\pi / 2$. Thus, for $N$ kink-antikink pairs we get $E_{k}^{N}=N \pi$, and the size $L_{k}^{N}$ of the array has to obey $L_{k}^{N}=2 N \pi$. 
The above kink or antikink is stable. We write $\phi(x, t)=\phi(x)+\sum_{n} \eta_{n}(x) \cos \left(w_{n} t\right)$ in order to obtain the Schrödinger-like equation for stability

$$
-\frac{d^{2} \eta_{n}}{d x^{2}}-\eta_{n}=w^{2} \eta_{n}
$$

The energies are such that $w_{n}=\sqrt{n(n+2)}$, and the eigenfunctions are even or odd, for $n$ even or odd, respectively. They are given by

$$
\eta_{n}(x)=\sqrt{2 / \pi} \begin{cases}\cos [(n+1) x] & \text { for } n=0,2,4, \ldots \\ \sin [(n+1) x] & \text { for } n=1,3,5, \ldots\end{cases}
$$

The gap $w_{n+1}-w_{n}$ starts at $\sqrt{3}$, and converges to 1 for increasing $n$. This spectrum is similar to the spectrum found in Ref. [19].

The case of positive pressure is different. This phase is unstable, and the energy of kink or antikink diverges. We illustrate this situation with the model

$$
V(\phi)=\frac{1}{2} \sec ^{2}(\phi)
$$

which is described by $W(\phi)=\ln [\sec (\phi)+\tan (\phi)]$ and requires that $\phi \in[-\pi / 2, \pi / 2]$. In this case the first-order equations are

$$
\frac{d \phi}{d x}= \pm \sec (\phi)
$$

These equations are solved by $\phi(x)= \pm \arcsin (x)$ for $x \in[-1,1]$. The derivative diverges for $x \rightarrow \pm 1$. Also, the energy density is $\varepsilon(x)=1 /\left(1-x^{2}\right)$, which diverges in the limit $x \rightarrow \pm 1$, leading to divergent energy. We also notice that $1 / \sqrt{1-x^{2}}$ would be the zero mode, but this is not normalizable in the interval $x \in[-1,1]$.

\section{Ending comments}

In this work we have modified the tachyon action (1.1) by changing the square root contribution as in (1.2). We have found stable, finite energy tachyon kinks in the case of vanishing pressure in several different models. We have also found a network of kinkantikink configurations in the case of negative pressure. Although the scenario one finds in the case of negative pressure is similar to other extensions that appeared recently, the case of vanishing pressure is new, and it supports regular, stable and finite energy tachyon kinks which nicely lend thenselves to Sen's singular solutions in the appropriate limit.

The modification that we introduce gives rise to another phase, corresponding to the case of positive pressure. However, the phase with positive pressure is unstable, giving rise to tachyon kink and antikink which end with divergent derivative, signalling the presence of divergent energy. The cases concerning positive and negative pressure have interesting analogies with field-theoretic models, which allowed illustrating both possibilities within simpler scenarios.

We thank F.A. Brito for discussions, and CAPES, CNPq, PROCAD/CAPES and PRONEX/FAPESQ/CNPq for partial support. 


\section{References}

[1] A. Sen, J. High Energy Phys. 08, 012 (1998); 09, 023 (1998)).

[2] P. Horava, Adv. Theor. Math. Phys. 2, 1373 (1999).

[3] A. Sen, J. High Energy Phys. 10, 008 (1999).

[4] M. Garousi, Nucl. Phys. B 584, 284 (2000).

[5] E. Bergshoeff et al. J. High Energy Phys. 05, 009 (2000).

[6] M. Garousi, Nucl. Phys. B 647, 117 (2002).

[7] D. Kutasov and V. Niarchos, Nucl. Phys. B 666, 56 (2003).

[8] A. Sen, Phys. Rev. D 68, 106003 (2003).

[9] A. Sen, Phys. Rev. D 68, 066008 (2003).

[10] E.J. Copeland, P.M. Saffin, and D.A. Steer, Phys. Rev. D 68, 065013 (2003).

[11] R. Hobart, Proc. Phys. Soc. Lond. 82, 201 (1963).

[12] G.H. Derrick, J. Math Phys. 5, 1252 (1964).

[13] R. Jackiw, Rev. Mod. Phys. 49, 681 (1977).

[14] D. Bazeia, J. Menezes, and R. Menezes, Phys. Rev. Lett. 91, 241601 (2003).

[15] D. Bazeia and F.A. Brito, Phys. Rev. D 61, 105019 (2000).

[16] C. Kim, Y. Kim, and C.O. Lee, JHEP 05, 020 (2003).

[17] Ph. Brax, J. Mourad, and D.A. Steer, Phys. Lett. B 575, 115 (2003).

[18] D. Bazeia, Phys. Rev. D 60, 067705 (1999).

[19] J.A. Minahan and B. Zwiebach, J. High Energy Phys. 03, 038 (2001). 